\title{
Sperm-associated antigen 9 promotes astrocytoma cell invasion through the upregulation of podocalyxin
}

\author{
JIAODE JIANG ${ }^{1}$, YUNSHENG LIU ${ }^{2}$, WENHUA FANG ${ }^{3}$ and FENG LIU ${ }^{1}$ \\ ${ }^{1}$ Department of Neurosurgery, The Third Xiangya Hospital; ${ }^{2}$ Department of Neurosurgery, \\ Xiangya Hospital, Central South University, Changsha, Hunan; ${ }^{3}$ Department of Neurosurgery, \\ The First Affiliated Hospital, Fujian Medical University, Fuzhou, Fujian, P.R. China
}

Received September 12, 2013; Accepted March 18, 2014

DOI: $10.3892 / \mathrm{mmr} .2014 .2168$

\begin{abstract}
Podocalyxin (PODXL) has been found to increase the aggressive phenotype of a number of cancers, including astrocytoma. In addition, the progression of astrocytoma has been associated with sperm-associated antigen 9 (SPAG9), a recently characterized oncoprotein. In the present study, the association between SPAG9 and PODXL in human astrocytoma invasion and the underlying mechanisms were investigated for the first time, to the best of our knowledge. Overexpression and knockdown of SPAG9 were performed in SW1783 (grade III astrocytoma) and U87 (grade IV astrocytoma; glioblastoma) cells, respectively. PODXL expression at both the mRNA and the protein level, as well as the PODXL gene promoter activity, were significantly increased and decreased in parallel with the overexpression and knockdown of SPAG9 in astrocytoma cells; these effects were blocked by the selective c-Jun N-terminal kinase (JNK) inhibitor SP600125 $(5 \mu \mathrm{M})$ and restored by the JNK agonist anisomycin $(25 \mathrm{ng} / \mathrm{ml})$, respectively. SPAG9 overexpression significantly increased cell invasion and matrix metalloproteinase-9 (MMP-9) expression in SW1783 cells, and this effect was reversed by knockdown of PODXL. In U87 cells, knockdown of SPAG9 markedly decreased cell invasion and MMP-9 expression, which was completely restored by overexpression of PODXL. In conclusion, it was demonstrated in the present study that SPAG9 upregulates PODXL expression in human astrocytoma cells at the PODXL gene promoter/transcriptional level through a JNK-dependent mechanism and that PODXL is a critical mediator of the promoting effect of SPAG9 on astrocytoma cell invasion, possibly through upregulation of MMP-9 expression. This study provides novel insights into the molecular mechanisms involved in astrocytoma invasion.
\end{abstract}

Correspondence to: Dr Feng Liu, Department of Neurosurgery, The Third Xiangya Hospital, Central South University, 138 Tongzipo Road, Changsha, Hunan 410013, P.R. China

E-mail: 59149033@qq.com

Key words: sperm-associated antigen 9, c-Jun N-terminal kinase, podocalyxin, astrocytoma, cell invasion, matrix metalloproteinase- 9

\section{Introduction}

Gliomas are the most common and devastating primary central nervous system neoplasms and account for $77 \%$ of all brain tumors (1). Gliomas are subdivided into oligodendrogliomas, ependymomas, astrocytomas and oligoastrocytomas based on the resemblance of tumor cells to the original parental cells. According to World Health Organization (WHO) classifications, astrocytomas are further classified into four clinical grades: Pilocytic astrocytomas (WHO grade I), diffuse astrocytomas (WHO grade II), anaplastic astrocytomas (WHO grade III) and glioblastoma multiforme (WHO grade IV) (2). In excess of 51,000 individuals are diagnosed with primary brain tumors in the United States each year, and $75 \%$ of those diagnosed with astrocytoma are likely to succumb within five years of diagnosis (3). Although the length of survival has been enhanced by surgery, radiation and chemotherapy, astrocytoma mortality remains high. The median survival of patients with glioblastoma is between 12 and 15 months and between two and five years for patients with anaplastic astrocytoma $(1,2)$. Therefore, novel strategies for the treatment of astrocytoma, particularly glioblastoma, are required. However, the mechanism underlying the malignant progression of astrocytomas has not yet been fully elucidated.

Sperm-associated antigen 9 (SPAG9) is one of a family of scaffolding proteins that selectively mediates c-Jun N-terminal kinase (JNK) signaling by aggregating specific components of the mitogen-activated protein kinase cascade to form a functional JNK signaling module (4). Previous studies have shown that SPAG9 is overexpressed in a number of human cancers, including renal, breast, thyroid, cervical and colon carcinomas (5-9). Furthermore, SPAG9-small interfering RNA treatment has been shown to inhibit tumor cell proliferation and invasion (5-9). A recent study demonstrated that SPAG9 was overexpressed in human astrocytomas and that SPAG9 depletion in astrocytoma cells inhibited cell invasion through downregulation of matrix metalloproteinase-9 (MMP-9) (10), suggesting that SPAG9 has an important role in astrocytoma invasion.

Podocalyxin (PODXL) is a transmembrane protein that is expressed by several types of human cells, including hematopoietic progenitors and vascular endothelial cells, as well as platelets (11). Increased PODXL expression has been associated with a subset of aggressive types of cancer, including acute 
myeloid and lymphoid leukemia, myeloid sarcomas and certain breast, liver, pancreatic and kidney tumors $(11,12)$. PODXL has been shown to lead to increased cell invasion and MMP expression in breast and prostate cancer cells (13). Furthermore, PODXL expression has been detected in $42.9 \%$ of anaplastic astrocytoma samples and $54.8 \%$ of glioblastoma samples, suggesting that PODXL is associated with the malignant progression of astrocytoma (14). A recent study showed that PODXL promotes cell invasion through the upregulation of MMP-9 expression in human astrocytoma cells (15), indicating that PODXL also has an important role in astrocytoma invasion.

In the present study, the association between SPAG9 and PODXL in human astrocytoma invasion and the underlying mechanisms involved were investigated for the first time, to the best of our knowledge.

\section{Materials and methods}

Cells lines, plasmids and reagents. SW1783 and U87 human astrocytoma cell lines were purchased from the American Type Culture Collection (Rockville, MD, USA). Human full-length SPAG9 and PODXL cDNAs were subcloned into pcDNA 3.1 expression vectors, respectively. Human PODXL promoter-luciferase reporter construct was generated as previously described (16). Briefly, a 1,528-bp DNA fragment of the 5 ' regulatory region of the human $P O D X L$ gene, comprising 1,297 bp upstream from the transcription start site plus $231 \mathrm{bp}$ of 5'-untranslated region, was amplified and inserted upstream of the luciferase gene. Human PODXL short hairpin RNA (shRNA) plasmid (RHS3979-98487921) and pLKO.1 empty plasmid (RHS4080) were purchased from Open Biosystems (Huntsville, AL, USA). Anti-PODXL (3D3) (39-3800) antibody was purchased from Invitrogen Life Technologies (Carlsbad, CA, USA). Human JIP-4/SPAG9-shRNA lentiviral particles (sc-62513-V), control shRNA lentiviral particles-A (sc-108080) and anti-MMP-9 (sc-13520) and anti-JIP-4/SPAG9 (sc-67649) antibodies were purchased from Santa Cruz Biotechnology, Inc. (Santa Cruz, CA, USA). SuperFect ${ }^{\text {TM }}$ transfection reagent was purchased from Qiagen (Valencia, CA, USA). A dual-luciferase reporter assay system was obtained from Promega Corporation (Madison, WI, USA). The selective JNK inhibitor SP600125, the JNK agonist anisomycin, puromycin, G418 and all chemicals of reagent grade were purchased from Sigma (St. Louis, MO, USA).

Transfection and lentiviral transduction. The SPAG9 and PODXL expression constructs were transfected into cells using SuperFect ${ }^{\mathrm{TM}}$ transfection reagent (Qiagen) in accordance with the manufacturer's instructions. Pools of stable transductants were generated via selection with G418 $(800 \mu \mathrm{g} / \mathrm{ml})$. Lentiviral transduction was performed and pools of stable transductants were generated via selection with puromycin $(5 \mu \mathrm{g} / \mathrm{ml})$.

Western blot analysis. Immunoblotting was performed using the respective antibodies. Briefly, cells were dissolved in $250 \mu \mathrm{l}$ 2X SDS loading buffer $(62.5 \mathrm{mM}$ Tris- $\mathrm{HCl}, \mathrm{pH} 6.8,2 \%$ SDS, $25 \%$ glycerol, $0.01 \%$ bromophenol blue and 5\% 2-mercaptoethanol) and incubated at $95^{\circ} \mathrm{C}$ for $10 \mathrm{~min}$. Equal quantities of proteins for each sample were separated using $10 \%$ SDS-PAGE and blotted onto a polyvinylidene difluoride microporous membrane (Millipore, Billerica, MA, USA). Membranes were incubated for $1 \mathrm{~h}$ with a $1 / 1,000$ dilution of primary antibody (1/10,000 for 3D3 PODXL blotting) and then washed and revealed using secondary antibodies with horseradish peroxidase conjugate (1/5,000; $1 \mathrm{~h}$; Santa Cruz Biotechnology, Inc.). Peroxidase was revealed using the GE Healthcare enhanced chemiluminescence kit (Piscataway, NJ, USA). Proteins were quantified prior to being loaded onto the gel. Expression of TIMP-1 and TIMP-2 was also examined with western blot analysis using antibodies (sc-365905 for TIMP-1 and sc-6835 for TIMP-2) from Santa Cruz Biotechnology, Inc.

Quantitative transcription polymerase chain reaction ( $q P C R$ ). RNA was prepared from cells using TRIzol ${ }^{\circledR}$ reagent followed by purification with TURBO DNA-free system (Ambion, Austin, TX, USA). The cDNAs were synthesized using SuperScript II reverse transcriptase (Invitrogen Life Technologies). qPCR was performed on the LightCycler thermal cycler system (Roche Diagnostics, Indianapolis, IN, USA) using a SYBR-Green I kit (Roche Diagnostics) in accordance with the manufacturer's instructions. The results were normalized against the housekeeping gene $G A P D H$ in the same sample. The primers used were as follows: $P O D X L$, 5'-AATTCCTTTCCCAGTTGT-3' (forward) and 5'-TTCTCA GTAAATTCCAGTGTA-3' (reverse); GAPDH, 5'-GACTCA TGACCACAGTCCATGC-3' (forward) and 5'-AGAGGC AGGGATGATGTTCTG-3' (reverse). Each experiment was repeated two times and performed in triplicate.

Luciferase assay. SW1783 and U87 cells were transfected with human $P O D X L$ promoter-luciferase reporter constructs using SuperFect ${ }^{\mathrm{TM}}$ transfection reagent (Qiagen). The plasmid pRL-CMV encoding Renilla reniformis luciferase (at 1/5 molar ratio to test plasmids) was co-transfected with test plasmids in each transfection as an internal control for data normalization. Luciferase assays were performed using a dual-luciferase reporter assay system (Promega Corporation) in accordance with the manufacturer's instructions. Each experiment was repeated three times and performed in duplicate.

In vitro cell invasion assay. Transwell ${ }^{\circledR}$ cell-culture chambers with $8-\mu \mathrm{m}$ pore size (BD Biosciences, Bedford, MA, USA) for 24-well plates were coated with $50 \mu 1$ Matrigel $^{\mathrm{TM}}(10 \mathrm{mg} / \mathrm{ml}$; BD Biosciences), which was diluted 1:3 in RPMI-1640. SW1783 and U87 cells were seeded in the upper chamber at $5 \times 10^{5}$ cells/well in RPMI-1640 serum-free medium. Complete medium $(600 \mathrm{ml})$ was added to the lower chamber. Cells were allowed to migrate for $24 \mathrm{~h}$ followed by fixation and staining with crystal violet. Invasion cells were counted in 10 random fields/chamber under the microscope. Each experiment was repeated three times and performed in triplicate.

Statistical analysis. Statistical analysis was performed using SPSS for Windows 10.0 (SPSS, Inc., Chicago, IL, USA). Data values are expressed as the mean \pm standard deviation. Comparisons of the means among multiple groups were performed using a one-way analysis of variance followed by post hoc pairwise comparisons using Tukey's tests. $\mathrm{P}<0.05$ was considered to indicate a statistically significant difference. 
A

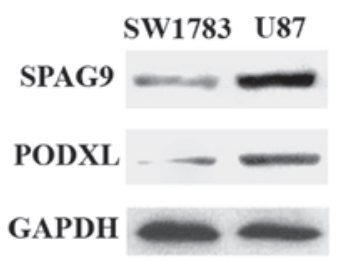

B

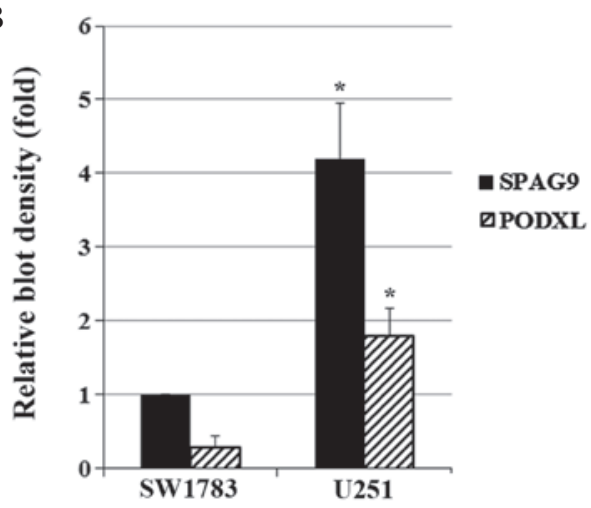

Figure 1. SPAG9 and PODXL expression in SW1783 and U87 astrocytoma cells. (A) SPAG9 and PODXL expression was analyzed using western blotting. GAPDH was used as a loading control. (B) Density of the SPAG9 and the PODXL blots was normalized against GAPDH to obtain the relative blot density, expressed as fold-change to the relative SPAG9 blot density of SW1783 cells (designated as 1). ${ }^{*} \mathrm{P}<0.05$, compared with SW1783 cells. SPAG9, sperm-associated antigen 9; PODXL, podocalyxin.

A

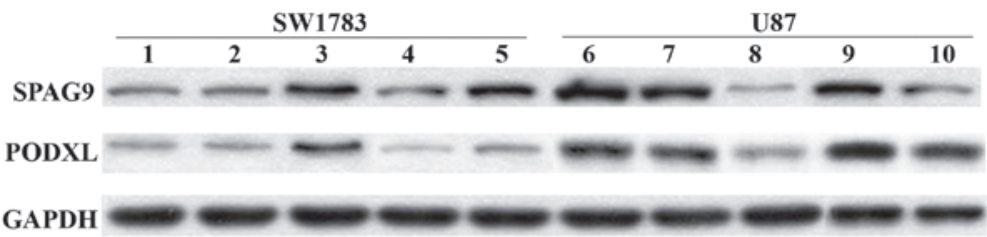

B

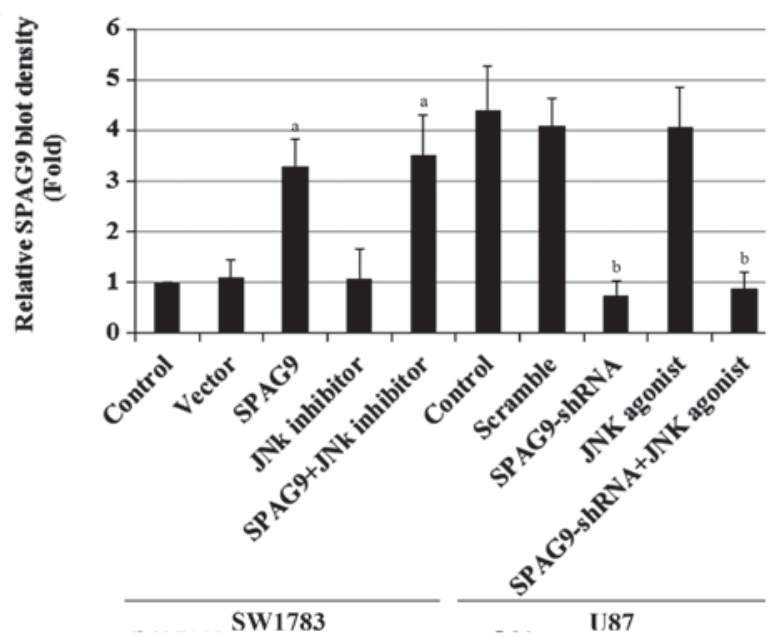

C

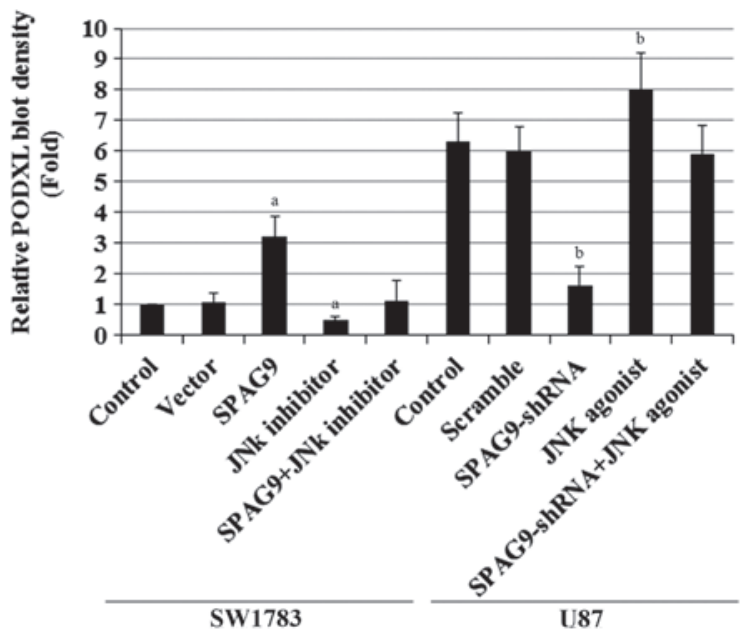

Figure 2. SPAG9 and podocalyxin PODXL expression in astrocytoma cells with overexpression and knockdown of SPAG9. (A) In SW1783 cells, expression of SPAG9 in SW1783 control cells, cells stably transfected with empty pcDNA3 vector (Vector) and cells stably transfected with SPAG9 with or without JNK inhibitor SP600125 treatment $(5 \mu \mathrm{M}, 24 \mathrm{~h})$ was analyzed using western blotting (lane 1, Control; lane 2, Vector; lane 3, SPAG9; lane 4, JNK inhibitor; lane 5 , SPAG9+JNK inhibitor). In U87 cells, the PODXL protein level in the U87 control cells, cells stably transduced with scramble control shRNA (Scramble) and cells stably transduced with $S P A G 9$-shRNA with or without JNK agonist anisomycin treatment $(25 \mathrm{ng} / \mathrm{ml}, 24 \mathrm{~h})$ was analyzed with western blot analysis (lane 6, Control; lane 7, Scramble; lane 8, SPAG9-shRNA; lane 9, JNK agonist; lane 10, SPAG9-shRNA+JNK agonist). GAPDH blotting was used as a loading control. Density of the (B) SPAG9 and (C) PODXL blots was normalized against GAPDH to obtain the relative blot density, expressed as fold-change to the relative (B) SPAG9 or (C) PODXL blot density of SW1783 control cells (designated as 1). In SW1783 cells, ${ }^{a}$ P $<0.05$ compared with Control and Vector. In U87 cells, ${ }^{b} \mathrm{P}<0.05$ compared with Control and Scramble. SPAG9, sperm-associated antigen 9; PODXL, podocalyxin; JNK, c-Jun N-terminal kinase; shRNA, short hairpin RNA.

\section{Results}

Effect of the overexpression and knockdown of SPAG9 on PODXL expression in human astrocytoma cells. As shown in Fig. 1, SW1783 (grade III astrocytoma) cells exhibited a relatively low constitutive expression of SPAG9 and PODXL compared with U87 (grade IV astrocytoma; glioblastoma) cells. Western blot analysis showed that stable transfection of SPAG9 led to SPAG9 overexpression in SW1783 cells, which was not affected by the JNK inhibitor SP600125 $(5 \mu \mathrm{M})$. However, knockdown of $S P A G 9$ by shRNA resulted in $>75 \%$ decrease in endogenous SPAG9 protein expression in U87 cells, which was not affected by the JNK agonist anisomycin (25 ng/ml) (Fig. 2). PODXL protein expression in SW1783 


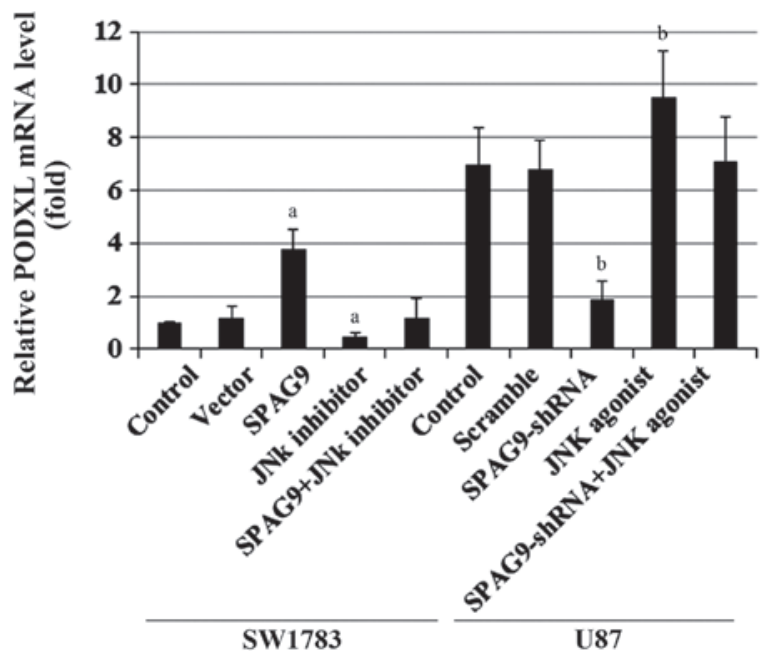

Figure 3. PODXL mRNA levels in astrocytoma cells with overexpression and knockdown of SPAG9. In SW1783 cells, the PODXL mRNA levels in SW1783 control cells, cells stably transfected with empty pcDNA3 vector (Vector) and cells stably transfected with SPAG9 with or without JNK inhibitor SP600125 treatment $(5 \mu \mathrm{M}, 24 \mathrm{~h})$ were analyzed using qPCR. In U87 cells, the PODXL mRNA levels in the U87 control cells, cells stably transduced with scramble control shRNA (Scramble) and cells stably transduced with SPAG9-shRNA with or without JNK agonist anisomycin treatment $(25 \mathrm{ng} / \mathrm{ml}, 24 \mathrm{~h})$ were analyzed using qPCR. PODXL mRNA levels are shown as fold-changes compared with SW1783 control cells (designated as 1). In SW1783 cells, ${ }^{\mathrm{a}} \mathrm{P}<0.05$ compared with Control and Vector. In U87 cells, ${ }^{\mathrm{b}} \mathrm{P}<0.05$ compared with Control and Scramble. SPAG9, sperm-associated antigen 9; PODXL, podocalyxin; JNK, c-Jun N-terminal kinase; shRNA, short hairpin RNA; qPCR, quantitative polymerase chain reaction

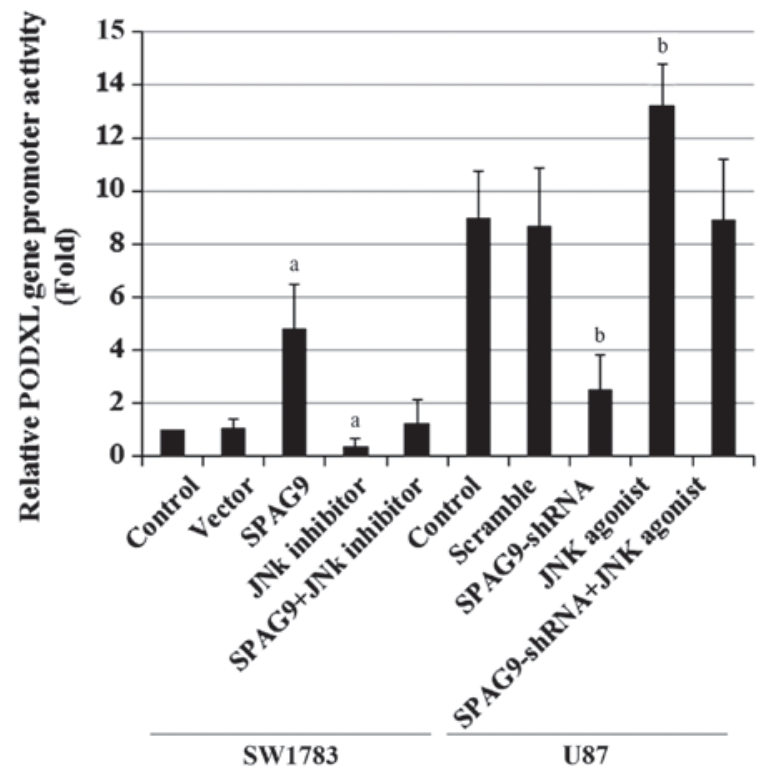

Figure 4. Effect of $S P A G 9$ on human $P O D X L$ promoter activity. SW1783 and U87 cells were transfected with human $P O D X L$ promoter-luciferase reporter plasmids. After $24 \mathrm{~h}$, luciferase assays were performed. In SW1783 cells, luciferase activities in the SW1783 control cells, cells stably transfected with empty pcDNA3 vector (Vector) and cells stably transfected with $S P A G 9$ with or without JNK inhibitor SP600125 treatment $(5 \mu \mathrm{M}, 24 \mathrm{~h})$ were analyzed. In U87 cells, luciferase activities in the U87 control cells, cells stably transduced with scramble control shRNA (Scramble) and cells stably transduced with SPAG9-shRNA with or without JNK agonist anisomycin treatment $(25 \mathrm{ng} / \mathrm{ml}$, $24 \mathrm{~h}$ ) were analyzed. Luciferase activities are expressed as fold-changes compared with SW1783 control cells (designated as 1). In SW1783 cells, ${ }^{\mathrm{a}} \mathrm{P}<0.05$, compared with Control and Vector. In U87 cells, ${ }^{\mathrm{b}} \mathrm{P}<0.05$, compared with Control and Scramble. SPAG9, sperm-associated antigen 9; PODXL, podocalyxin; JNK, c-Jun N-terminal kinase; shRNA, short hairpin RNA. cells increased in parallel with $S P A G 9$ overexpression, and this was inhibited by SP600125. In U87 cells, PODXL protein expression decreased in parallel with SPAG9-knockdown, and this was rescued by anisomycin (Fig. 3). Similar data trends were observed with PODXL mRNA expression in SW1783 and U87 cells (Fig. 3).

Effect of the overexpression and knockdown of SPAG9 on $P O D X L$ promoter activity in human astrocytoma cells. To determine whether SPAG9 regulates PODXL expression in human astrocytoma cells by altering the PODXL gene promoter activity, SW1783 and U87 cells were transfected with human $P O D X L$ promoter-luciferase reporter plasmids. Luciferase assays showed that the PODXL gene promoter activity in SW1783 cells was increased by SPAG 9 overexpression, and this effect was inhibited by SP600125 $(5 \mu \mathrm{M})$. In U87 cells, the $P O D X L$ gene promoter activity was decreased by $S P A G 9$-knockdown, while activity was completely restored by anisomycin ( $25 \mathrm{ng} / \mathrm{ml})$ (Fig. 4).

Functional role of PODXL in SPAG9-enhanced cell invasion and MMP-9 expression in human astrocytoma cells. SPAG9 and PODXL have been suggested to promote cell invasion through the upregulation of MMP-9 expression in human astrocytoma cells $(10,15)$. Since the above findings showed that SPAG9 expression may affect PODXL expression in human astrocytoma cells, the functional role of PODXL in SPAG9-enhanced cell invasion and MMP-9 expression in astrocytoma cells was then examined. In vitro cell invasion assays showed that SPAG9 overexpression significantly increased cell invasion in SW1783 cells, and that this effect was reversed by PODXL-knockdown (Fig. 5). In U87 cells, knockdown of $S P A G 9$ markedly decreased cell invasion, and this decrease was completely restored by overexpression of $P O D X L$. Similar data trends were observed with MMP-9 protein expression in SW1783 and U87 cells (Fig. 6). By contrast, the expression of tissue inhibitor of metalloproteinase 1 (TIMP1) and TIMP2 was not changed by overexpression or knockdown of either SPAG9 or PODXL (data not shown).

\section{Discussion}

PODXL has been found to increase the aggressive phenotype of a number of cancers, including astrocytoma (15). In addition, the progression of astrocytoma, as well as that of a number of other human malignancies, has been associated with SPAG9, a recently characterized oncoprotein (10). The present study provides the first evidence, to the best of our knowledge, that SPAG9 upregulates PODXL expression in human astrocytoma cells and that a major part of the promoting effect of SPAG9 on astrocytoma cell invasion is mediated by PODXL, most likely through the upregulation of MMP-9 expression.

SPAG9 is overexpressed in $60 \%$ of human astrocytomas and is associated with higher tumor grades. In the present study, several human astrocytoma cell lines were assessed and it was found that, while high levels of SPAG9 were expressed in U87 cells, SPAG9 was expressed at a relatively low constitutive level in SW1783 cells. Thus, knockdown and overexpression of $S P A G 9$ were respectively performed in the two cell lines in the context of the study aims. 


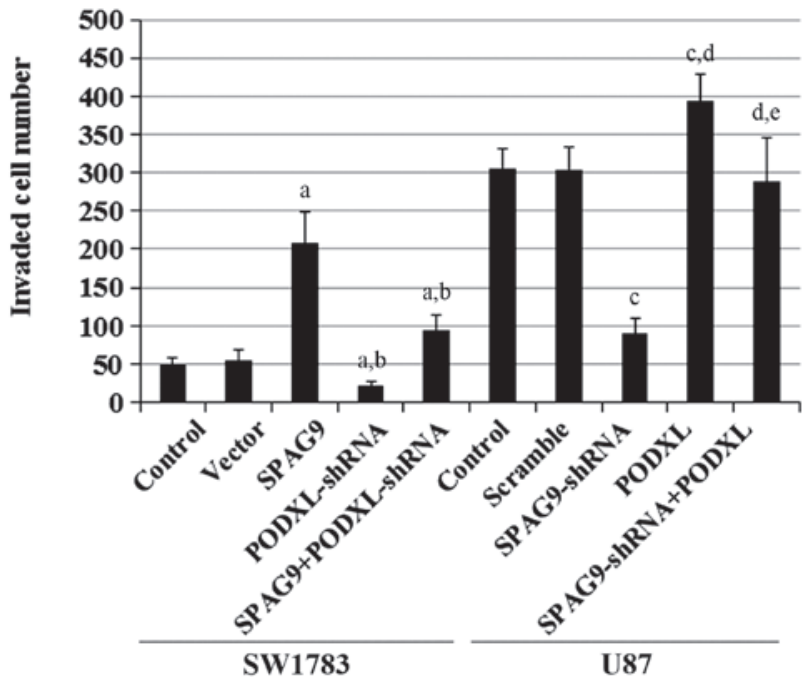

Figure 5. In vitro cell invasion in astrocytoma cells with overexpression and knockdown of SPAG9 and/or PODXL. In SW1783 cells, in vitro cell invasion assays were performed in SW1783 control cells, cells stably transfected with empty pcDNA3 vector (Vector), cells stably transfected with $S P A G 9$, cells stably transduced with $P O D X L$-shRNA and cells stably expressing SPAG9 and PODXL-shRNA. In U87 cells, in vitro cell invasion assays were performed in U87 control cells, cells stably transduced with scramble control shRNA (Scramble), cells stably transduced with $S P A G$-shRNA, cells stably transfected with $P O D X L$ and cells stably expressing $S P A G 9$-shRNA and PODXL. Invaded cell numbers were counted. In SW1783 cells, ${ }^{a} \mathrm{P}<0.05$ compared with Control and Vector; ${ }^{\mathrm{b}} \mathrm{P}<0.05$ compared with SPAG9. In U87 cells, ${ }^{\circ} \mathrm{P}<0.05$ compared with Control and Scramble; ${ }^{\mathrm{d}} \mathrm{P}<0.05$ compared with SPAG9-shRNA; ${ }^{\mathrm{P}}<0.05$ compared with PODXL. SPAG9, sperm-associated antigen 9; PODXL, podocalyxin; shRNA, short hairpin RNA.

SPAG9 has been suggested to be involved in the activation of the JNK pathway, and JNK signaling is considered to be involved in SPAG9-enhanced tumor cell invasion (10). In the present study, PODXL expression at the mRNA and protein levels was significantly increased and decreased in parallel with the overexpression and knockdown, respectively, of SPAG9 in astrocytoma cells, and these effects were blocked by the selective JNK inhibitor and restored by the JNK agonist, respectively. The results suggest that SPAG9 expression may affect PODXL expression in human astrocytoma cells at the gene transcription level through a JNK-dependent mechanism. Luciferase assays confirmed that SPAG9 may enhance PODXL gene promoter/transcriptional activities in astrocytoma cells through a JNK-dependent mechanism. However, how SPAG9 modulates $P O D X L$ promoter activities has yet to be elucidated and requires further analysis.

It has been shown that SPAG9 in human astrocytoma cells promotes cell invasion, possibly through the regulation of MMP-9 transcription (10). A recent study has shown that PODXL promotes cell invasion through upregulating MMP-9 expression in human astrocytoma cells (15). This suggests that the interaction between SPAG9 and PODXL may be important in promoting astrocytoma cell invasion. The results from the present study suggested that PODXL is a downstream effector of SPAG9/JNK signaling; therefore, the functional role of PODXL in SPAG9-enhanced astrocytoma cell invasion and MMP-9 expression was investigated. It was found that $P O D X L$-knockdown almost eliminated the increased cell invasion and MMP-9 expression caused

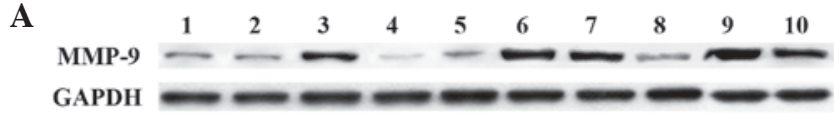

B

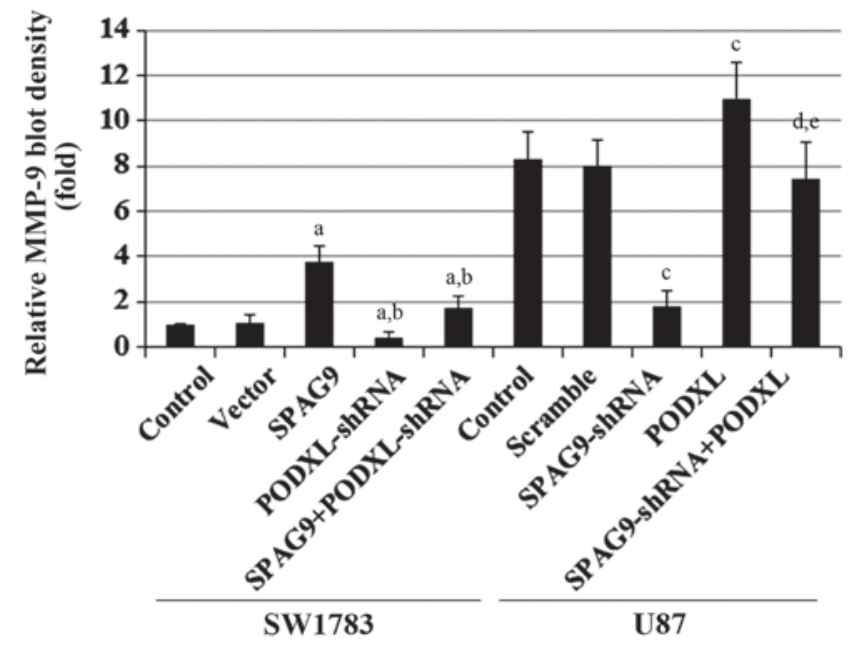

Figure 6. MMP-9 expression in astrocytoma cells with overexpression and knockdown of SPAG9 and/or PODXL. (A) In SW1783 cells, MMP-9 expression was determined in SW1783 control cells, cells stably transfected with empty pcDNA3 vector (Vector), cells stably transfected with SPAG9, cells stably transduced with $P O D X L$-shRNA and cells stably expressing SPAG9 and $P O D X L$-shRNA (lanes 1-5, respectively). In U87 cells, MMP-9 expression was determined in U87 control cells, cells stably transduced with scramble control shRNA (Scramble), cells stably transduced with SPAG-shRNA, cells stably transfected with PODXL and cells stably expressing both SPAG9-shRNA and PODXL (lanes 6-10, respectively). GAPDH was used as a loading control. (B) Density of the MMP-9 blot was normalized against GAPDH to obtain the relative MMP-9 expression, which is expressed as fold-change to the relative MMP-9 blot density of SW1783 control cells (designated as 1). In SW1783 cells, ${ }^{\text {aP }}<0.05$ compared with Control and Vector; ${ }^{\mathrm{b}} \mathrm{P}<0.05$ compared with SPAG9. In U87 cells, ${ }^{\mathrm{c}} \mathrm{P}<0.05$ compared with Control and Scramble; ${ }^{\mathrm{d}} \mathrm{P}<0.05$ compared with $S P A G 9$-shRNA; ${ }^{\mathrm{e}} \mathrm{P}<0.05$ compared with PODXL. MMP-9, matrix metalloproteinase-9; SPAG9, sperm-associated antigen 9; PODXL, podocalyxin; JNK, c-Jun N-terminal kinase; shRNA, short hairpin RNA.

by SPAG9 overexpression in SW1783 cells, while PODXL overexpression completely restored the decreased cell invasion and MMP-9 expression caused by SPAG9-knockdown in U87 cells. The results indicate that PODXL is a critical mediator of the promoting effect of SPAG9 on astrocytoma cell invasion, most likely through the upregulation of MMP-9 expression. Consistent with a previous study (15), TIMP1 and TIMP2 were not found to be involved in the SPAG9/PODXL/astrocytoma cell invasion process.

In conclusion, it was demonstrated in the present study that SPAG9 upregulates PODXL expression in human astrocytoma cells at the PODXL gene promoter/transcriptional level through a JNK-dependent mechanism and that PODXL is a critical mediator of the promoting effect of SPAG9 on astrocytoma cell invasion, most likely through the upregulation of MMP-9 expression. This study provides novel insights into the molecular mechanisms of astrocytoma invasion.

\section{Acknowledgements}

This study was supported by Hunan Provincial Natural Science Foundation (grant nos. 12H5438 and 13A1116), Hunan, China. 


\section{References}

1. Ohgaki $\mathrm{H}$ and Kleihues P: Genetic alterations and signaling pathways in the evolution of gliomas. Cancer Sci 100: 2235-2241, 2009.

2. Louis DN, Ohgaki H, Wiestler OD, et al: The 2007 WHO classification of tumours of the central nervous system. Acta Neuropathol 114: 97-109, 2007.

3. Giese A, Bjerkvig R, Berens ME and Westphal M: Cost of migration: invasion of malignant gliomas and implications for treatment. J Clin Oncol 21: 1624-1636, 2003.

4. Ando K, Uemura K, Kuzuya A, et al: N-cadherin regulates p38 MAPK signaling via association with JNK-associated leucine zipper protein: implications for neurodegeneration in Alzheimer disease. J Biol Chem 286: 7619-7628, 2011.

5. KanojiaD, Garg M, Gupta S, Gupta A and Suri A: Sperm-associated antigen 9, a novel biomarker for early detection of breast cancer. Cancer Epidemiol Biomarkers Prev 18: 630-639, 2009.

6. Garg M, Kanojia D, Suri S and Suri A: Small interfering RNAmediated down-regulation of SPAG9 inhibits cervical tumor growth. Cancer 115: 5688-5699, 2009.

7. Garg M, Kanojia D, Salhan S, et al: Sperm-associated antigen 9 is a biomarker for early cervical carcinoma. Cancer 115: 2671-2683 2009.

8. Garg M, Kanojia D, Suri S, Gupta S, Gupta A and Suri A: Sperm-associated antigen 9: a novel diagnostic marker for thyroid cancer. J Clin Endocrinol Metab 94: 4613-4618, 2009.
9. Kanojia D, Garg M, Gupta S, Gupta A and Suri A: Sperm-associated antigen 9 is a novel biomarker for colorectal cancer and is involved in tumor growth and tumorigenicity. Am J Pathol 178: 1009-1020, 2011.

10. Yi F, Ni W, Liu W, et al: SPAG9 is overexpressed in human astrocytoma and promotes cell proliferation and invasion. Tumour Biol 34: 2849-2855, 2013.

11. Nielsen JS and McNagny KM: The role of podocalyxin in health and disease. J Am Soc Nephrol 20: 1669-1676, 2009.

12. Riccioni R, Calzolari A, Biffoni M, et al: Podocalyxin is expressed in normal and leukemic monocytes. Blood Cells Mol Dis 37: 218-225, 2006.

13. Sizemore S, Cicek M, Sizemore N, Ng KP and Casey G: Podocalyxin increases the aggressive phenotype of breast and prostate cancer cells in vitro through its interaction with ezrin. Cancer Res 67: 6183-6191, 2007.

14. Hayatsu N, Kaneko MK, Mishima K, Nishikawa R, Matsutani M, Price JE and Kato Y: Podocalyxin expression in malignant astrocytic tumors. Biochem Biophys Res Commun 374: 394-398, 2008.

15. Wu H, Yang L, Liao D, Chen Y, Wang W and Fang J: Podocalyxin regulates astrocytoma cell invasion and survival against temozolomide. Exp Ther Med 5: 1025-1029, 2013.

16. Butta N, Larrucea S, Alonso S, et al: Role of transcription factor $\mathrm{Sp} 1$ and $\mathrm{CpG}$ methylation on the regulation of the human podocalyxin gene promoter. BMC Mol Biol 7: 17, 2006. 